\title{
Correction to: Real-World Switching to Riociguat: Management and Practicalities in Patients with PAH and CTEPH
}

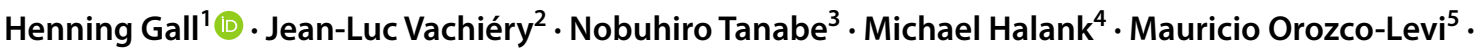 \\ Lisa Mielniczuk ${ }^{6} \cdot$ MiKyung Chang $^{7} \cdot$ Kai Vogtländer $^{8} \cdot$ Ekkehard Grünig $^{9}$
}

Published online: 3 April 2018

(c) The Author(s) 2018

\section{Correction to: Lung \\ https://doi.org/10.1007/s00408-018-0100-3}

The original version of this article unfortunately contained a mistake. In the "Results" section, the percentage of patients with inoperable or persistent/recurrent CTEPH included in the study was reported as $85 \%$. This has been corrected to $68 \%$ with this erratum.

The original article can be found online at https://doi.org/10.1007/ s00408-018-0100-3.

Henning Gall

Henning.Gall@innere.med.uni-giessen.de

1 Department of Internal Medicine, Justus-Liebig-University Giessen, Universities of Giessen and Marburg Lung Center (UGMLC), Member of the German Center for Lung Research (DZL), Klinikstrasse 32, 35392 Giessen, Germany

2 Département de Cardiologie, Cliniques Universitaires de Bruxelles, Hôpital Erasme, Brussels, Belgium

3 Department of Respirology and Department of Advanced Medicine in Pulmonary Hypertension, Graduate School of Medicine, Chiba University, Chiba, Japan

4 Medical Clinic 1/Pneumology, University Hospital Carl Gustav Carus, Dresden, Germany

5 Hospital Internacional de Colombia, Fundación Cardiovascular de Colombia, Santander, Colombia

6 University of Ottawa Heart Institute, Ottawa, ON, Canada

7 Bayer AG, Berlin, Germany

8 Bayer AG, Wuppertal, Germany

9 Center for Pulmonary Hypertension, Thorax Clinic at the University Hospital, Heidelberg, Germany 